\title{
Synthesis and Luminescence Properties of the Binary/Ternary Complex of Samarium Ion with Phenacylmethyl P-Tolyl Sulfoxide and Benzoic Acid
}

\author{
Kui-Suo Yang ${ }^{1}$, Wen-Xian $\mathrm{Li}^{1}{ }^{*}$, and Jin-Rong $\mathrm{Bao}^{1}$ \\ ${ }^{1}$ College of Chemistry and Chemical Engineering, Inner Mongolia University, Hohhot 010021, \\ People's Republic of China
}

\begin{abstract}
A new ligand phenacylmethyl p-tolyl sulfoxide (denoted as $\mathrm{L}_{1}$ ) has been synthesized by an asymmetric synthesis method. Its novel binary complex $\mathrm{Sm}\left(\mathrm{L}_{1}\right)_{7} \cdot\left(\mathrm{ClO}_{4}\right)_{3} \cdot \mathrm{H}_{2} \mathrm{O}$, and ternary complex $\mathrm{Sm}\left(\mathrm{L}_{1}\right)_{6} \cdot\left(\mathrm{L}_{2}\right) \cdot\left(\mathrm{ClO}_{4}\right)_{2} \cdot 6 \mathrm{H}_{2} \mathrm{O}$ (benzoic acid denoted as $\mathrm{L}_{2}$ ) have been synthesized. The structure and composition of the samarium complexes characterized by elemental analysis, molar conductivity, IR, UV, and ${ }^{1}$ HNMR. Additionally, fluorescence spectra measurements shows that the complexes display stronger fluorescence intensity in solid state, indicating the ligands could efficiently transfer the energy to $\mathrm{Sm}^{3+}$ ions in the complexes. The ternary complex has stronger fluorescence intensity than the binary complex.
\end{abstract}

\section{Introduction}

Rare earth complexes have attracted much attention for a wide variety of applications in the fields of lighting devices, and biomedical analysis $[1,2]$. There are numerous examples of luminescent rare earth complexes reported in the literature [3,4]. As is known, the intra-configuration $4 \mathrm{f} \rightarrow 4 \mathrm{f}$ transitions in rare earth ions are forbidden, so the luminescence intensity of the $\mathrm{RE}^{3+}$ ions are weakening [5]. The organic ligands with great conjugated action the as sensitizers coordinated with the $\mathrm{RE}^{3+}$ which can transfer the energy to it effectively and further to increase its luminescence intensity. The organic ligands that exhibited this property were called as "antennas" [6]. In the past several decades, many sulfoxide complexes had been synthesized and intensively studied due to the superb coordination ability of the sulfoxide ligand [7,8]. Europium and terbium complexes are studied a lot. There are few researches about samarium complexes. So it is extremely vital significant for the comprehensive utilization of $\mathrm{Sm}^{3+}$ to research the luminescence properties of the samarium complexes.

Thus, we have synthesized a phenacylmethyl p-tolyl sulfoxide $\left(\mathrm{L}_{1}\right)$. Furthermore, the $\mathrm{L}_{1}$ as the first ligand and benzoic acid $\left(\mathrm{L}_{2}\right)$ as the second ligand coordinated with samarium ions in the paper. The luminescence properties of as-synthesized binary and ternary

\footnotetext{
*Corresponding author: nmglwx@163.com
} 
samarium complexes were studied. The luminescence study indicates that ternary samarium complex has higher fluorescence intensity than binary samarium complex.

\section{Experimental details}

\subsection{Materials and physical measurements.}

$\mathrm{Sm}_{2} \mathrm{O}_{3}$ (purity $>99.99 \%$ ) was supplied by Beijing Chemical Reagent Company. The $\mathrm{Sm}$ (III) perchlorate was prepared from dissolving in $2 \mathrm{~mol} \cdot \mathrm{L}^{-1} \mathrm{HClO}_{4}$, and then evaporated and dried in vacuum. All other chemicals were analytical grade and were used as received without further purification. Elemental analysis was carried out on a HANAU analyzer. Conductivity measurement was made on a DJS-1 conductivity meter. The infrared spectra were determined on a Nicolet NEXUS-670 FT-IR spectrophotometer. The ultraviolent spectral were recorded on a TU-1901 double beam spectrophotometer. ${ }^{1} \mathrm{HNMR}$ spectra were measured on Bruker AC-500 spectrometer in DMSO- $\mathrm{d}_{6}$. Fluorescence spectra and fluorescent lifetime were determined on a FLS920 with the slit width of $1 \mathrm{~nm}$.

\subsection{Preparation of the europium ternary complex.}

The $\mathrm{L}_{1}$ was prepared by oxidation of $\mathrm{C}_{6} \mathrm{H}_{5} \mathrm{COCH}_{2} \mathrm{SC}_{6} \mathrm{H}_{4} \mathrm{CH}_{3}$ according to the literature [9]. The appropriate concentration of $\mathrm{L}_{1}$ anhydrous ethanol solution was added into samarium perchlorate anhydrous ethanol solution under magnetic stirring for $1.0 \mathrm{~h}$. A white precipitate appeared, the white precipitate was separated by suction filtration, purified by washing with anhydrous ether for several times. Finally, white powder was obtained. The preparation of binary samarium complex was similar to the ternary samarium complex.

\section{Results and discussions}

\subsection{Composition analysis.}

The elemental analysis and molar conductivity data of samarium complexes showed in Table 1 . The $\lambda \mathrm{m}$ value of the binary complex in DMF is in good accordance with the formula as 1:3 electrolyte and the $\lambda \mathrm{m}$ value of the ternary complex in DMF is in good accordance with the formula as 1:2 electrolyte [10]. According to the elemental analysis, the compositions of the binary and the ternary complexes are suggested as $\mathrm{Sm}\left(\mathrm{L}_{1}\right)_{7} \cdot\left(\mathrm{ClO}_{4}\right)_{3} \cdot 6 \mathrm{H}_{2} \mathrm{O}$ and $\mathrm{Sm}\left(\mathrm{L}_{1}\right)_{6} \cdot\left(\mathrm{L}_{2}\right) \cdot\left(\mathrm{ClO}_{4}\right)_{2} \cdot 6 \mathrm{H}_{2} \mathrm{O}$, respectively.

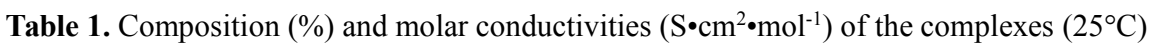

\begin{tabular}{|c|c|c|c|c|c|}
\hline Complexes & $\mathrm{M}$ & \multicolumn{3}{|c|}{ Anal. calcd. (found) (\%) } & $\lambda_{\mathrm{m}}$ \\
\hline & & $\mathrm{C}$ & $\mathrm{H}$ & $\mathrm{Sm}$ & \\
\hline $\mathrm{Sm}\left(\mathrm{L}_{1}\right)_{7} \cdot\left(\mathrm{ClO}_{4}\right)_{3} \cdot 6 \mathrm{H}_{2} \mathrm{O}$ & 2363.33 & $53.31(53.14)$ & $4.69(4.38)$ & $6.43(6.72)$ & 213 \\
\hline $\mathrm{Sm}\left(\mathrm{L}_{1}\right)_{6} \cdot\left(\mathrm{L}_{2}\right) \cdot\left(\mathrm{ClO}_{4}\right)_{2} \cdot 6 \mathrm{H}_{2} \mathrm{O}$ & 2127.34 & $54.72(54.77)$ & $4.78(4.45)$ & $7.14(6.91)$ & 183 \\
\hline
\end{tabular}

\section{2 ${ }^{1}$ HNMR spectra analysis.}

The ${ }^{1} \mathrm{HNMR}$ of $\mathrm{L}_{1}$ shows that there are multiple resonance peaks for two phenyl groups at $\delta 7.375-7.980 \mathrm{ppm}$, and the integral intensity illustrates it contains nine protons. The proton resonances peaks of one methylene and one methyl group are at $\delta 4.685-4.747 \mathrm{ppm}$ and $\delta$ 
2.370-2.504 ppm, which have two and three proton respectively (Fig. 1a). The result showed that we have synthesized the $\mathrm{L}_{1}$ successfully and the purity of the obtained ligand is supernal. The ${ }^{1} \mathrm{HNMR}$ spectra of the samarium complexes are similar as that of $\mathrm{L}_{1}$, which have shifted at different degree (Fig.1b,c). The reason may be concerned with coordinate effect.
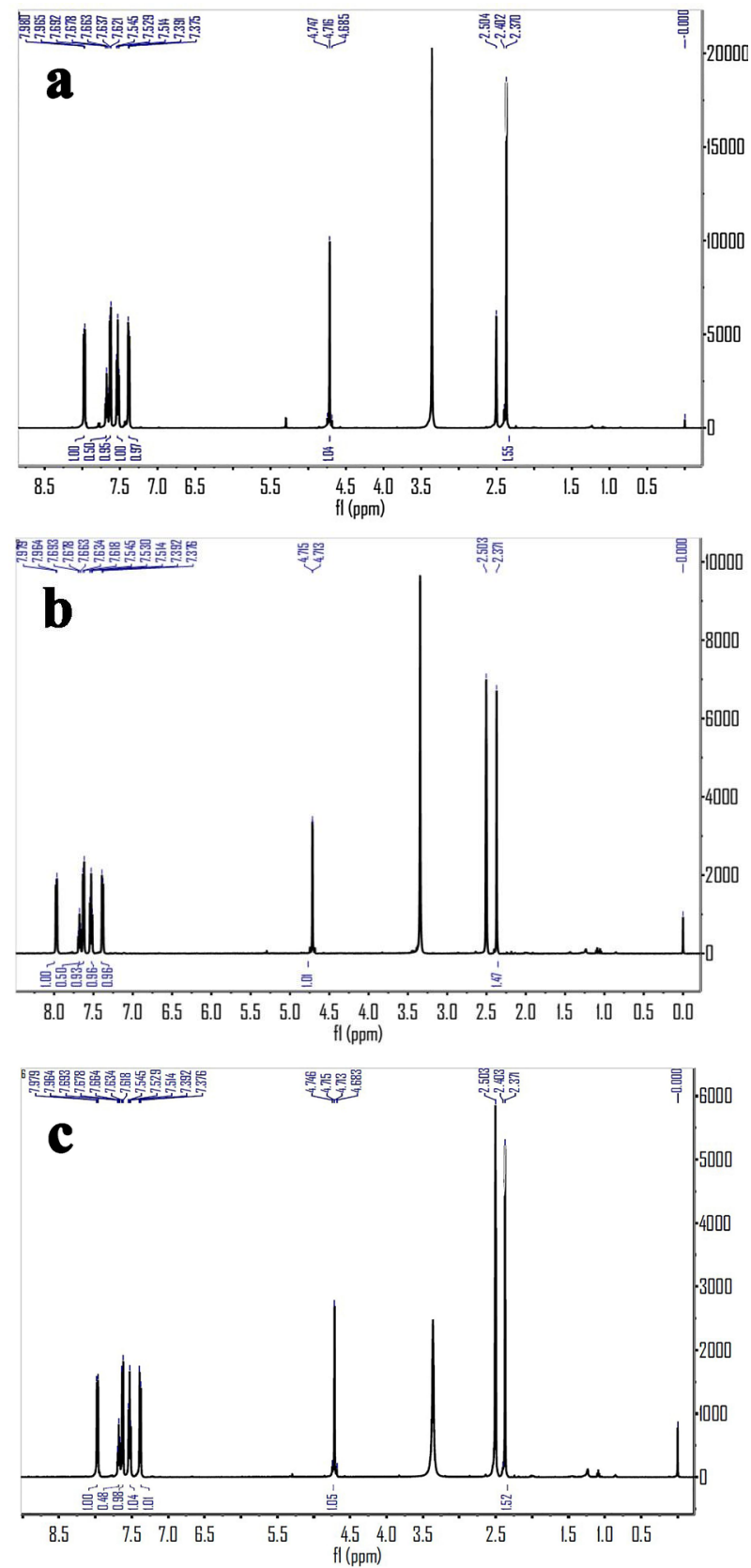

Fig. 1. The ${ }^{1} \mathrm{HNMR}$ spectrum of $\mathrm{a} . \mathrm{L}_{1} ; \mathrm{b}$. binary samarium complex; c. ternary samarium complex 


\subsection{Infrared spectra analysis.}

Fig. 2a show the IR spectrum of $\mathrm{L}_{1}$, the stretching vibration absorption of sulflnyl group appeared at $1049 \mathrm{~cm}^{-1}\left(v_{\mathrm{S}=0}\right)$. It shifts to $982 \mathrm{~cm}^{-1}$ and $979 \mathrm{~cm}^{-1}$ in IR spectra of binary and ternary samarium complexes (Fig. 2c,d), respectively, which suggests that $\mathrm{Sm}^{3+}$ ions are bonded with the oxygen atom of the sulflnyl group. Whereas the carbonyl group stretching vibration absorption in IR spectrum of $\mathrm{L}_{1}$ (Fig. 2a) appears at $1677 \mathrm{~cm}^{-1}\left(v_{\mathrm{C}=0}\right)$, no significant shift is found in IR spectra of binary and ternary systems (Fig. 2c,d), which is an evidence that the oxygen atom in carbonyl group is not coordinated with $\mathrm{Sm}^{3+}$ ion. The $v_{\mathrm{as}}\left(\mathrm{COO}^{-}\right)$and $v_{\mathrm{s}}\left(\mathrm{COO}^{-}\right)$absorption of $\mathrm{L}_{2}$ occurred at $1550 \mathrm{~cm}^{-1}$ and $1416 \mathrm{~cm}^{-1}$, and the $\Delta v$ value is $134 \mathrm{~cm}^{-1}$ (Fig. 2b). The $v_{\text {as }}\left(\mathrm{COO}^{-}\right)$and $v_{\mathrm{s}}\left(\mathrm{COO}^{-}\right)$absorption bands appear at 1641 $\mathrm{cm}^{-1}$ and $1335 \mathrm{~cm}^{-1}$ in the ternary samarium complex and the $\Delta v$ value was $306 \mathrm{~cm}^{-1}$ larger than that of $\mathrm{L}_{2}$ (Fig. 2d), which can be deduced that carboxyl group is coordinated with $\mathrm{Sm}^{3+}$ ion by monodentate type [11]. In terms of molar conductivities, it can be suggested that none of $\mathrm{ClO}_{4}^{-}$is coordinated with $\mathrm{Sm}^{3+}$ ions.
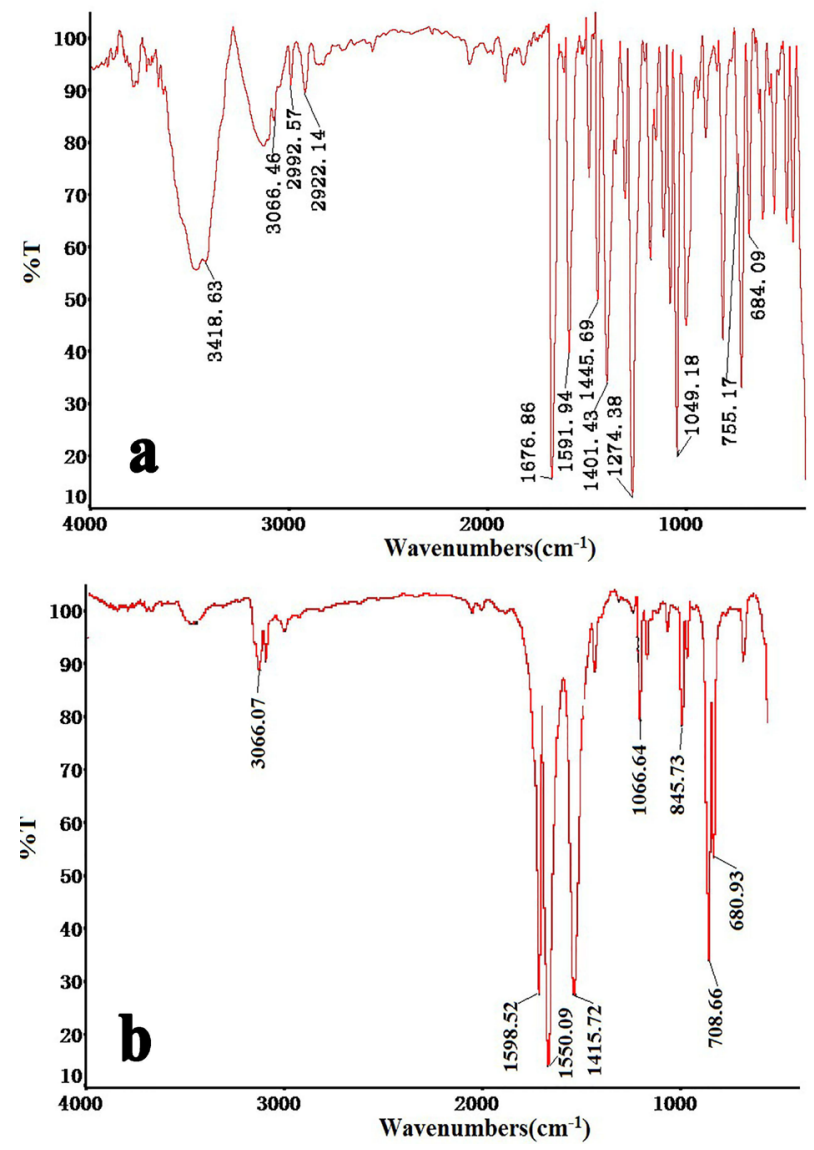


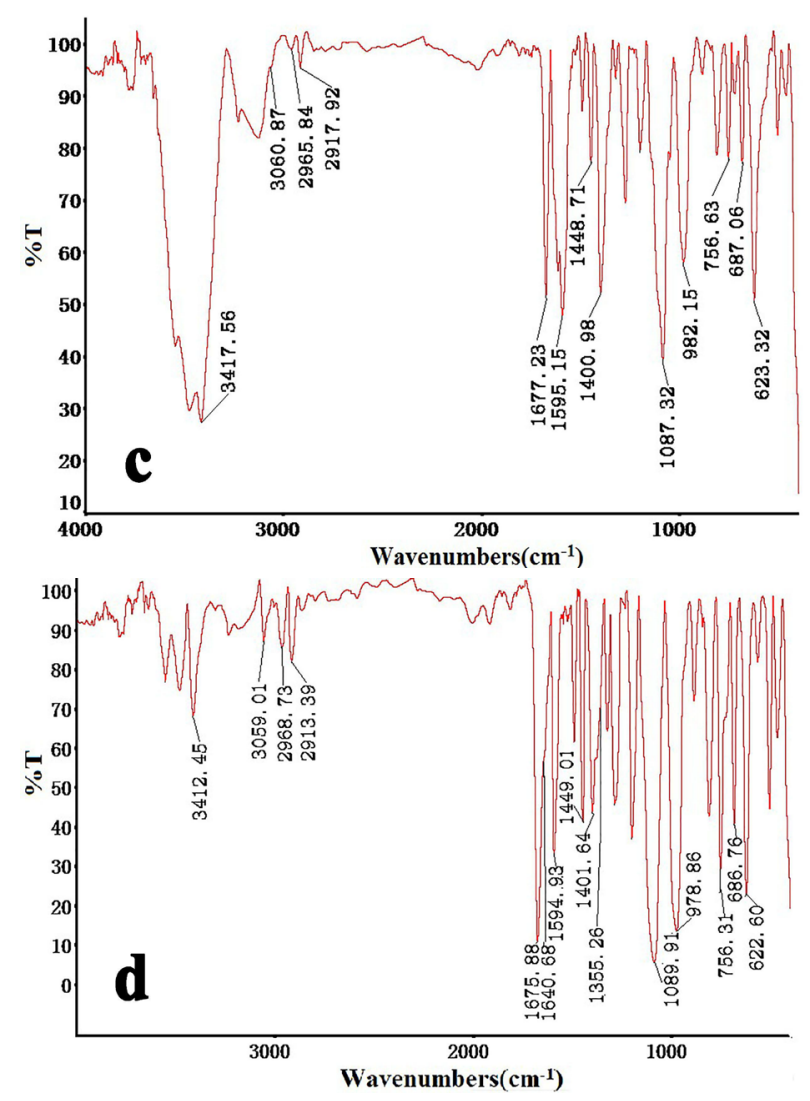

Fig. 2 Infrared absorption spectra of a. $\mathrm{L}_{1}$; b. $\mathrm{L}_{2}$; c. binary samarium complex; d. ternary samarium complex

\subsection{UV spectra analysis.}

The UV spectra of ligands displayed in Fig. $3 a$ and $b$, the major absorption bands of $\pi-\pi^{*}$ electronic transition in phenyl group are observed at $284 \mathrm{~nm}$ and $272 \mathrm{~nm}$, respectively. A strong absorption also observed at $284 \mathrm{~nm}$ and $285 \mathrm{~nm}$ for the binary and ternary samarium complexes, respectively (Fig. 3c,d), Which is no shift happening compared with the $\mathrm{L}_{1}$ (Fig. $3 b$ ). While the absorption band of binary samarium complex is red shift comparing to the absorption of $\mathrm{L}_{2}$ absorption band, from $272 \mathrm{~nm}$ to $285 \mathrm{~nm}$. It is found that the major absorption bands of the ligands still appeared after the formation of the complexes. 

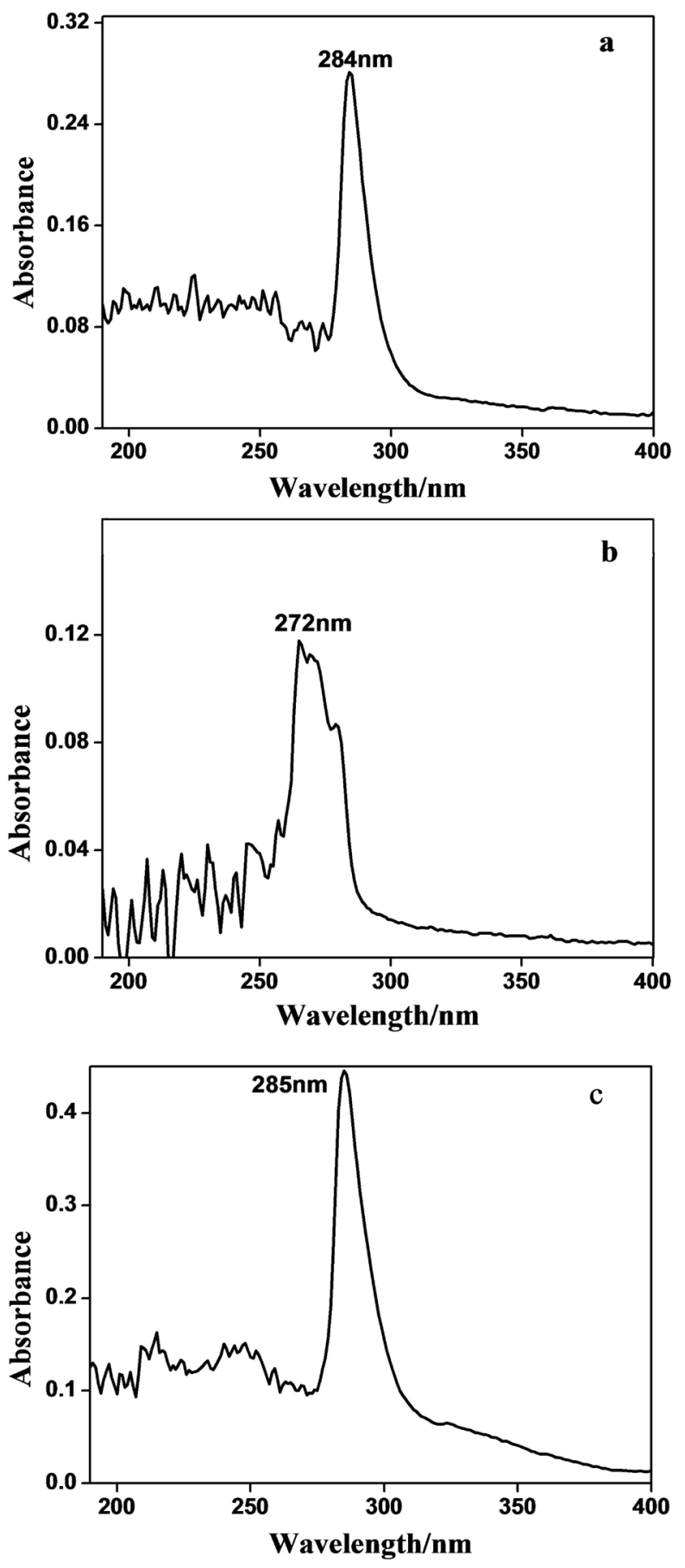


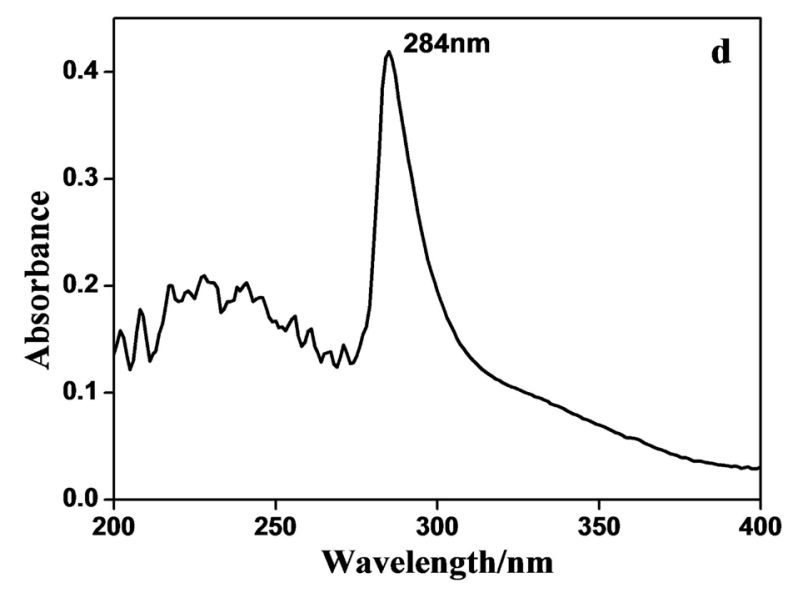

Fig. 3 UV absorption spectra of a. $L_{1} ;$ b. $L_{2} ;$ c. binary samarium complex; d. ternary samarium complex.

\subsection{Luminescence properties.}

Fig. 4 shows the excitation and emission spectra of binary samarium complex (a) and ternary samarium complex (b). The excitation spectra of the complexes were obtained by monitoring the emission of the complexes at the wavelength of $596 \mathrm{~nm}$. The maximum peak of binary complex was at $317 \mathrm{~nm}$ and ternary complex was at $322 \mathrm{~nm}$. The excitation peaks of the two complexes are similar. The emission peaks of the binary and ternary samarium complexes centered at 561,596 and $643 \mathrm{~nm}$, which are assigned to the transitions of ${ }^{4} \mathrm{G}_{5 / 2} \rightarrow{ }^{6} \mathrm{H}_{5 / 2},{ }^{4} \mathrm{G}_{5 / 2} \rightarrow{ }^{6} \mathrm{H}_{5 / 2},{ }^{4} \mathrm{G}_{5 / 2} \rightarrow{ }^{6} \mathrm{H}_{9 / 2}$, respectively. The binary and ternary samarium complexes display stronger emission intensity, indicating the ligands could efficiently transfer the energy to $\mathrm{Sm}^{3+}$ ions in the complexes. The strongest emission intensity of the ternary complex (19680 a.u) is 1.37 times as great as that of the binary complex (14330 a.u.). The result illustrate that the second ligand $\mathrm{L}_{2}$ can play a synergistic effect for the energy transfer process between the first ligand and $\mathrm{Sm}^{3+}$ ion.

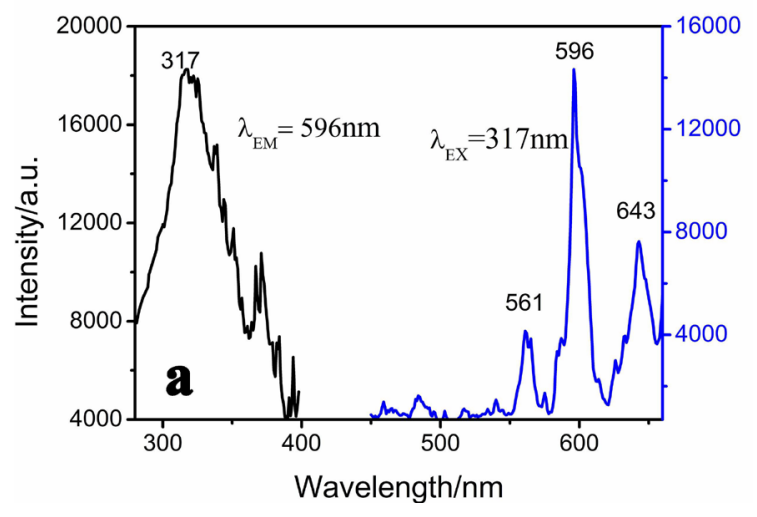




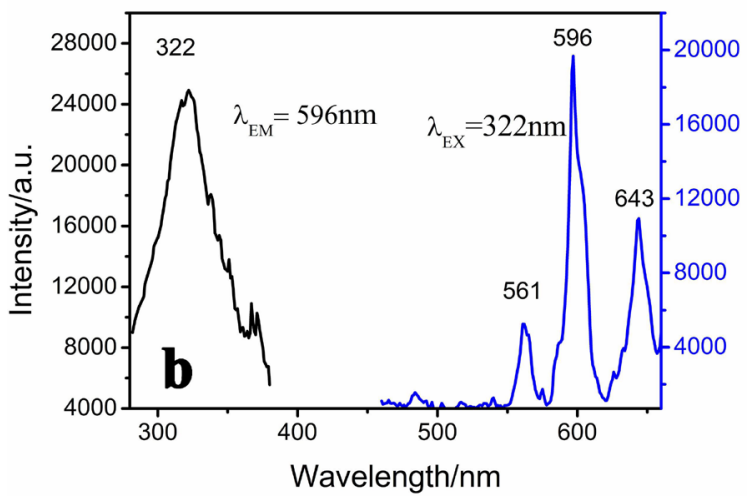

Fig. 4 Fluorescent EX and EM spectrum of a. binary samarium complex; b. ternary samarium complex

Fig. 5 shows the fluorescence decay curves and fit curves of the binary and ternary samarium complexes, respectively. The fluorescence lifetime value of samarium complexes were calculated by the double exponential mode. The lifetime of the ternary samarium complex is determined to be $65.19 \mu \mathrm{s}$, which is 1.46 times comparing to that of the binary samarium complex $44.46 \mu \mathrm{s}$.

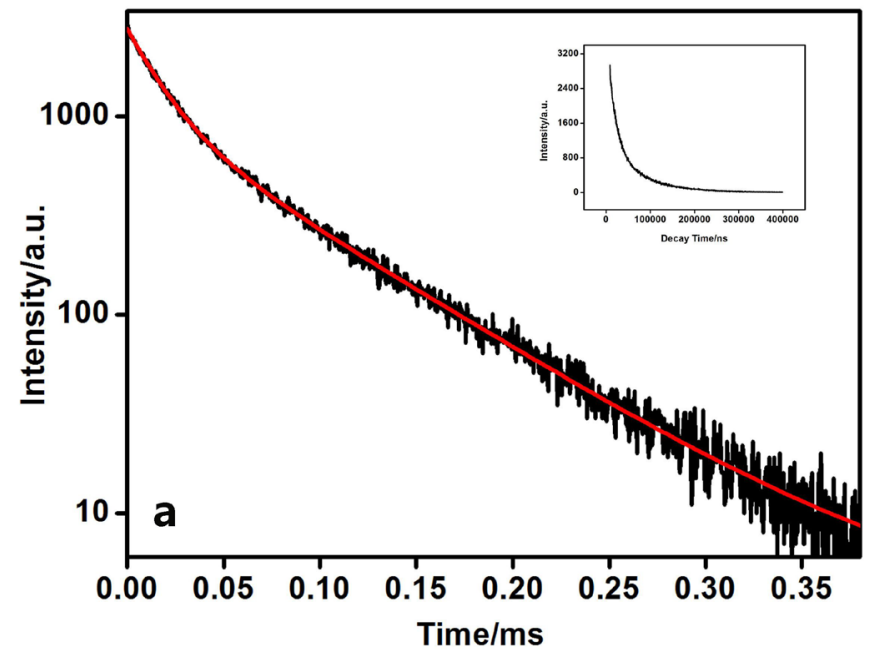




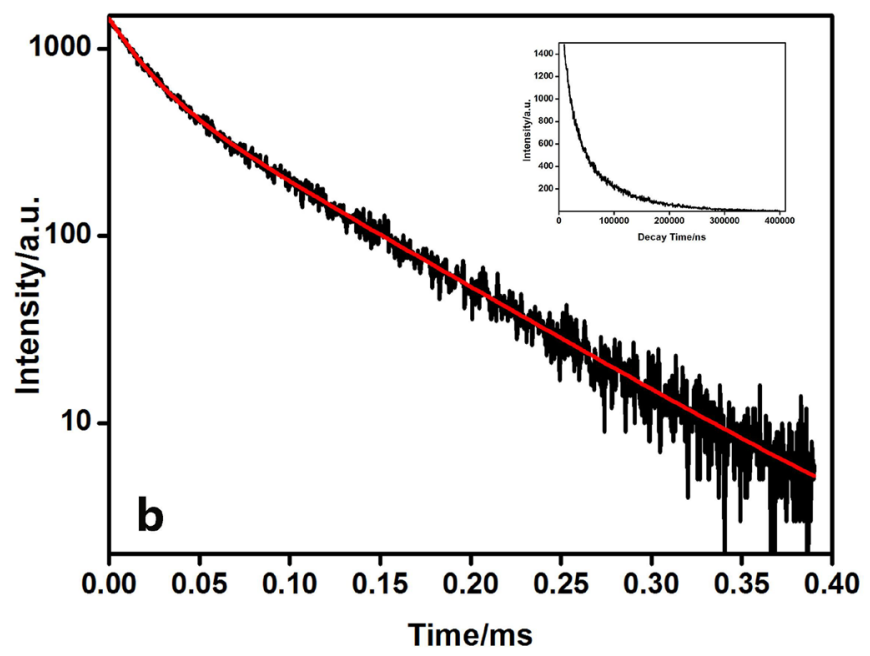

Fig. 5. Linear Fit Curves of a. binary samarium complex; b. ternary samarium complex (Inset: decay curve).

\section{Conclusions}

In this paper, a new ligand phenacylmethyl p-tolyl sulfoxide has been synthesized by an asymmetric synthesis method. The binary and ternary samarium complexes with phenacylmethyl p-tolyl sulfoxide as first ligand and benzoic acid as second ligand have been synthesized. The samarium complexes display preferable luminescence properties. The emission intensity of the ternary complex is 1.37 times as great as that of the binary complex, and the ternary complex has longer lifetime.

This work is supported by the Major projects of Natural Science Foundations of Inner Mongolia Science Foundation (2015ZD01) and the Natural Science Foundations of Inner Mongolia Science Foundation (2015MS0502).

\section{References}

1. A.P. Duarte, M. Gressier, M.J. Menu, et. al, J. Phys. Chem. C. 116 505-515(2012).

2. L.R. Yang, S. Song, H.M. Zhang, et. al, Synth. Met. 162 1775-1788(2012).

3. J.H. Xue, X.H. Hua, L.M. Yang, et. al, J. Mol. Struct. 1052 93-101(2013).

4. A. Gerus, K. Ślepokura, J. Lisowski, Inorg. Chem. 52 12450-12460(2013).

5. R. Gregory, D.R. Peterman, Coord. Chem. Rev. 174 283-299(1998).

6. J.M. Lehn, Angew. Chem. Int. Ed. 29 1304-19(1990).

7. W.X. Li, Y.S. Zheng, X.J. Sun, et. al, J. Fluoresc. 20 235-241(2010).

8. W.X. Li, Y.S. Zheng, L.H. Saiji, et. al, J. Lumin. 134 847-852(2013).

9. C. Cardellicchio, O.H. Omar., F. Naso, et. al, Tetrahedron: Asymmetry. 17:223-229(2006).

10. W.J. Greary, Coord. Chem. Rev. 7 81-122(1971).

11. G.B. Deacon, R.J. Phillips, Coord. Chem. Rev. 33 227-250(1980). 